\title{
Impact of Neighbor Awareness at the MAC Layer in a Vehicular Ad-hoc NETwork (VANET)
}

\author{
Marthinus J. Booysen ${ }^{\dagger}$, Sherali Zeadally ${ }^{\ddagger}$, and Gert-Jan van Rooyen ${ }^{\dagger}$ \\ ${ }^{\dagger}$ Department of Electrical and Electronic Engineering and the MIH Media Lab, Stellenbosch University \\ Stellenbosch, South Africa. Email: \{mjbooysen, gvrooyen\}@sun.ac.za \\ $\ddagger$ Department of Computer Science and Information Technology, University of the District of Columbia, \\ Washington DC, USA. Email: szeadally@udc.edu
}

\begin{abstract}
Despite various proposed Medium Access Control (MAC) approaches, efficient medium access in VANET remains a significant challenge, especially for the broadcast of safety messages. A key contributing factor to packet loss is the hidden terminal problem, which is exacerbated by the high node mobility apparent in VANET. Since the hidden terminal problem is fundamentally a problem of lack of awareness, this work evaluates the effects of increased neighbor awareness at the MAC layer. An increased awareness of neighboring nodes' slot allocation in a TDMA-based MAC, directly impacts on the number of available slots. Therefore, the effects of TDMA frame size is also evaluated for different awareness ranges. To support the analysis, a TDMA-based MAC with configurable neighbor awareness and configurable TDMA frame size is introduced. The impact is assessed using the packet delivery ratio, receiver throughput, and end-to-end latency. The results show packet delivery ratio and receiver throughput to increase for increased awareness ranges, up to an optimal of 2 hops, after which performance worsens. An increase in TDMA frame size leads to an increase in packet delivery ratio for small awareness ranges, and an oscillating increase for large awareness ranges. The receiver throughput also initially increases for an increased TDMA frame size, but reaches an optimum,also at 2 hops.
\end{abstract}

Index Terms-Vehicular Ad-hoc NETwork (VANET), Medium Access Control (MAC), Neighbor awareness.

\section{INTRODUCTION}

The field of vehicular communications has attracted substantial attention over the last few years. Vehicular Adhoc NETworks (VANETs) enable service delivery to vehicles by enabling Vehicle-to-Vehicle (V2V) and Vehicle-toInfrastructure (V2I) communications. In V2V scenarios, vehicles do not rely on infrastructure support to coordinate communications. The various applications proposed for VANET can be categorized as safety-related, traffic management, and service-related. A key challenge for Vehicular Ad-hoc NETworks (VANETs) is access control to the shared wireless medium, which is exacerbated by severe mobility, varying communications environments, and variable node densities and node distributions apparent in the vehicular environment [1], [2]. The requirements imposed by each application category are different, but all three applications categories rely on broadcasting [3].

This work focuses on Medium Access Control (MAC) performance in the broadcasting of safety messages in the $\mathrm{V} 2 \mathrm{~V}$ context. Safety messages need to be broadcast expediently, and with a high probability of successful reception [4]. Successful reception cannot, however, be evaluated in isolation, since a balance needs to be struck between the ratio of successful delivery and the volume of data transmitted. Transmission of safety messages therefore requires the MAC layer to transmit messages with a high packet delivery ratio, low end-to-end latency, and high receiver throughput.

One of the principal obstacles to efficient MAC layer communications in VANET is the hidden terminal problem [1], [5]. The hidden terminal problem describes a scenario where two nodes are both in range of a third node, but out of range of one another, leading to packet collisions (i.e. reducing packet delivery ratio) at the third node. The hidden terminal problem is also evident in static networks, or networks with low mobility, but is compounded by the high mobility evident in VANET. In essence, the hidden terminal problem is one of limited awareness. Various attempts have been made at addressing the hidden terminal problem, which implies an improvement in neighbor awareness at the MAC layer.

This paper evaluates the impact on MAC performance of changing the awareness range to overcome the hidden node problem. A Time Division Multiple Access (TDMA) MAC will be used to perform the evaluation. Since the neighbor awareness range is constrained by the available time slots in the TDMA frame, the effect of TDMA frame size on MAC is also investigated.

The rest of this paper is organized as follows. In section II we describe recent MAC approaches for VANETs. Section III presents the concept of neighbor awareness range used in this work. Section IV presents the simulation environments, including the MAC protocol used, the simulation software and configuration, and the performance metrics used in our performance evaluation tests. Performance results are presented in Section V. Finally, section VI concludes the paper.

\section{RELATED WORK}

The IEEE has standardized a contention-based MAC, called IEEE 802.11p, which has become the de facto standard MAC for use in VANETs [6]. Coordination in the broadcasting mode of IEEE 802.11p is based on Carrier Sense (CS): If a unit wants to broadcast, it waits for a period called the Arbitration Inter-Frame Space (AIFS). If no activity is sensed during the AIFS, the the node can transmit. However, if activity is sensed during the AIFS, the node that wants to 
transmit backs off for a period called the Contention Window $(\mathrm{CW})$, after which the process starts again with the AIFS. The CW is based on a counter, which increases if the CW is entered into again before a successful transmission attempt. In the unicast mode, IEEE $802.11 \mathrm{p}$ also requires nodes to use Request To Send/Clear To Send (RTS/CTS) handshaking before transmission is attempted, to overcome the hidden node problem.

Various MAC approaches have recently been proposed as alternatives to IEEE $802.11 \mathrm{p}$ [7]-[12]. The majority of alternative approaches are contention-free and use TDMA to coordinate access to the wireless medium. Since infrastructure cannot be assumed to be available in the $\mathrm{V} 2 \mathrm{~V}$ scenario, MAC methods need to be self-organizing (this is an inherent feature of the contention-based IEEE 802.11p). Two methods of selforganization are used by contention-free MAC approaches, namely a group-based approach [7]-[9] and a distributed approach [10]-[12].

In group-based approaches, a leader node coordinates communication and wireless access. The leader node needs to be aware of all the nodes in the group and the group is dependent on the continued in-range presence of the leader node, which is problematic in VANET since vehicles are highly mobile and have intermittent connectivity.

With distributed approaches, the coordination responsibility is shared among all the nodes in the network. One popular example is the Self-organizing TDMA-based (SoTDMA) MAC method proposed by Bilstrup et al. in [10] for safety critical message delivery. The method is presently employed in aviation and naval surveillance as part of the Automatic Identification System (AIS) and VHF Data Link Mode 4 respectively. In all the distributed approaches nodes frequently broadcast slot allocation information to notify their neighbors of their own, and in some cases, other nodes' slot selection. The method of notification and what is contained in the shared information is different for different MAC approaches, and could range from a binary bit for each transmission slot to indicate slot availability [11], to the full identification numbers of the nodes owning the slots [12]. The slot allocation information that each node transmits is based on information the node gathers from neighboring nodes. What constitutes neighboring nodes is different for different approaches and ranges from only neighbors in RF range (immediate neighbors) to 1-hop neighbors (immediate neighbors of immediate neighbors), or even accumulation with limitless number of hops [12], [13].

Both the group and distributed approaches incur communications overheads and suffer from implementation complexity to maintain coordination despite node mobility.

\section{NeIGHBor AwARENESS IN THE VANET MAC}

This section introduces the concept of awareness range in the MAC layer in VANET. In this paper, awareness range is used to describe the number of hops used to communicate slot allocation information between surrounding nodes, and is not strictly a distance. The awareness range therefore defines which nodes fall into the neighboring nodes category, in terms

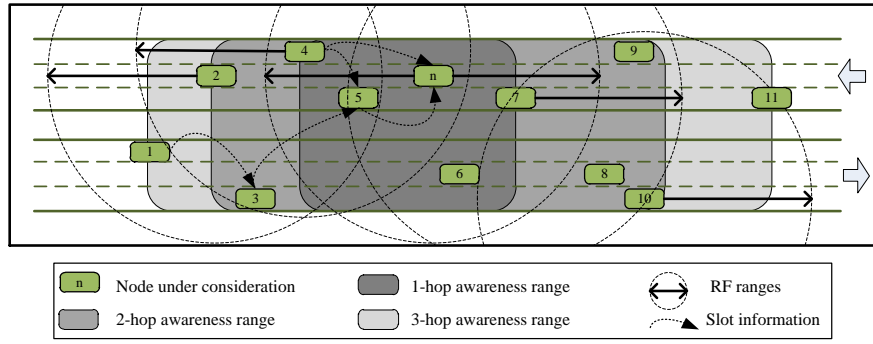

Fig. 1: Multi-hop awareness ranges.

of number of hops. The awareness range then determines to what hop count threshold the slot allocation information of each slot should be propagated.

The method of using multiple hops to transmit information further than the immediate RF range is not novel, and has been used extensively at the network layer. However, for multihop communication at the network layer, packets are passed on from a host node to one or more destination nodes, and not modified and combined with other packets to collectively organize medium access. For this work, multiple hops are used to convey slot allocation information at the MAC layer. For the multiple-hop transmission at the MAC layer, the information flow is from all nodes to all other nodes that are within a number of hops, i.e. within the awareness range. The slot allocation information is not merely passed on as packets, but combined with the slot allocation from surrounding nodes, and then shared in a collaborative attempt to better share the medium and avoid packet collisions. Therefore, the information flow of slot allocation is into the transmitting node, rather then away from it.

The nodes in RF range of a transmitting node are in the 0 -hop awareness range, since no hops are required to convey the information. For example, for node $n$ in Fig. 1, nodes 4, 5 , 6 , and 7 are all in RF range, and therefore define the 0 hop range. If the nodes in RF range of the transmitting node also convey the slot allocation information of only their RF neighbors to the transmitting node, the transmitting node will have a 1-hop awareness range. For example, for node $n$ in Fig. 1, slot allocation information from nodes 2 and 3 will have to hop once, thereby defining the 1-hop range. Similarly, nodes 1 and 11 define the 2-hop range. At each hop, slot allocation information from other nodes are appended, and only propagated if the slot allocation information is below the hop count threshold. It is possible that the same node could be in the 1-hop range through one route, and in the 2-hop route through another, e.g., node 4, which is both 1-hop and 2-hops away from node $n$ in Fig. 1 .

Contention-based MAC design assumes that nodes that are in range of one another can take steps, such as handshaking or carrier sensing, to coordinate communications at the MAC layer. The CTS/RTS handshaking required by IEEE $802.11 \mathrm{p}$ amounts to a 1-hop awareness range, since the receiving immediate neighbor only sends an RTS if the transmission does not clash with its own immediate neighbors' intentions. 
In the broadcasting mode, the CS only approach implies a 0hop awareness range, since the awareness range is equal to the communications range - which makes broadcasting with IEEE 802.11p especially susceptible to the hidden node problem.

The same assumption is made in the design of contentionfree MAC methods - coordination is performed between nodes in $\mathrm{RF}$ range, i.e. with awareness range equal to 0-hop, although some explicitly introduce a 1-hop awareness range to attempt to overcome the hidden terminal problem.

\section{Simulation Setup and Performance Metrics}

\section{A. Configurable TDMA-based Contention-Free MAC}

In this section we describe the self-organizing MAC approach that is used to demonstrate the effects on MAC performance of awareness range (number of hops) and TDMA frame size (number of slots). The MAC approach is TDMAbased and similar to RR-ALOHA+ [13], and uses multi-hop awareness to accumulate and distribute slot information from surrounding nodes.

Every node maintains its own Slot Allocation Table (SAT), to keep track of is neighbors' slot ownership. The SAT stored in each node is populated with the identification numbers of the nodes that own the slots. To control multi-hop awareness in the MAC layer, a hop counter is stored with each identifying number in the SAT, to indicate how many hops away the owning node is. Every node transmits its own SAT (its own version of slot ownership and distance) when it transmits in the slot it has assumed ownership of.

Three interdependent, concurrent, and competing processes run in every node, namely SAT Association, SAT Accumulation, and SAT Refresh.

SAT Association: When a node is turned on, it does not own a slot, and only selects an empty slot, after listening for a full TDMA cycle. The same process is entered if a slot conflict is detected.

SAT Accumulation describes the process of a node filling its own SAT with the slot allocations of nodes within its awareness range. For each packet that is received, the SAT information contained in the packet is evaluated, slot by slot, for possible incorporation into the local SAT. The hop count relating to each slot is checked against a configured threshold to limit the number of hops slot information can travel. This threshold sets the awareness range that is used in the system. The SAT Accumulation process gives precedence to slot information from nodes that are fewer hops away when filling the SAT. The hop count is therefore also used to evaluate whether the slot ownership information is of a nearer (i.e. fewer hops away) node than already contained in the local SAT, otherwise the received information for the slot is discarded. To reduce the probability of collisions further, a node relinquishes ownership of its own slot if any node in the awareness range claims the same slot, resulting in a partial refreshing (by means of overwriting) of the SAT.

To ensure that the SAT is not filled with outdated information, a SAT cleaning process (called SAT Refresh) is used, by which a slot is marked as unused, if no other nodes reported it as used in a TDMA cycle.

For the tests where no neighbor awareness is used, the three processes are replaced by a simple neighbor-agnostic algorithm. At the beginning of every TDMA frame, the node selects a transmission slot at random to transmit from. All slots are equally likely to be selected.

\section{B. Simulation Environment}

To accurately simulate communications in a vehicular environment, both the movement of vehicles and communications characteristics (such as signal path loss, receiver sensitivity, transmission power, etc.) need to be taken into account. The OMNeT++ network simulator is used to simulate the wireless communications network. Real-world maps from OpenStreetMap and a road traffic simulation package, called Simulation of Urban MObility (SUMO) is used to simulate mobility. The simulation tools are linked using Veins, proposed by Sommer et. al. in [14]. We conducted performance evaluation tests for highway traffic scenarios, with a vehicle density of 25 vehicles $/$ lane $/ \mathrm{km}$, and three lanes in each direction.

Table I lists some of the network parameters used in the network simulations.

TABLE I: Simulation parameters used in the simulations.

\begin{tabular}{|l|c|c|l|}
\hline Parameter & Value & Unit & Source \\
\hline Carrier frequency & 5.9 & $\mathrm{GHz}$ & {$[6]$} \\
Transmit power & $100.0(20)$ & $\mathrm{mW}(\mathrm{dBm})$ & {$[6][1]$} \\
Bitrate & 10 & $\mathrm{Mbps}$ & {$[12][6]$} \\
Packet size & 8 & $\mathrm{kbits}$ & {$[6]$} \\
Path loss exponent $(\alpha)$ & 3.0 & - & {$[15][16]$} \\
Receiver sensitivity & -80 & $\mathrm{dBm}$ & {$[1]$} \\
Contention Window & $31-1023$ & & {$[6][1]$} \\
Simulation time & 200 & seconds & \\
\hline
\end{tabular}

\section{Performance Metrics}

Three metrics are used to evaluate performance of MAC methods for the delivery of safety messages. The first metric is the packet delivery ratio, which measures the success ratio of transmissions. The ratio of received packets to transmitted packets are normalized to the number of in-range neighbors $\left(n_{\mathrm{RF}}\right)$ because in the case where no collisions occur during broadcast, all the in-range nodes will receive the transmission and result in $n_{\mathrm{RF}}$ receptions per transmission. The value of $n_{\mathrm{RF}}$ was approximated from a geometric model of the road environment and the vehicle density, and confirmed by setting the TDMA frame size to 1000 , and only allowing every tenth node to transmit, which diminishes interference.

The second metric we use is receiver throughput (the accumulated data successfully received in the network at the application layer per second). The receiver throughput is normalized to the average number of active vehicles in the network, to enable direct comparisons between scenarios with different road traffic densities or lengths.

The third performance metric is the end-to-end latency, which is defined as the delay from the application-layer of the transmitting node, to the application layer of the receiving 


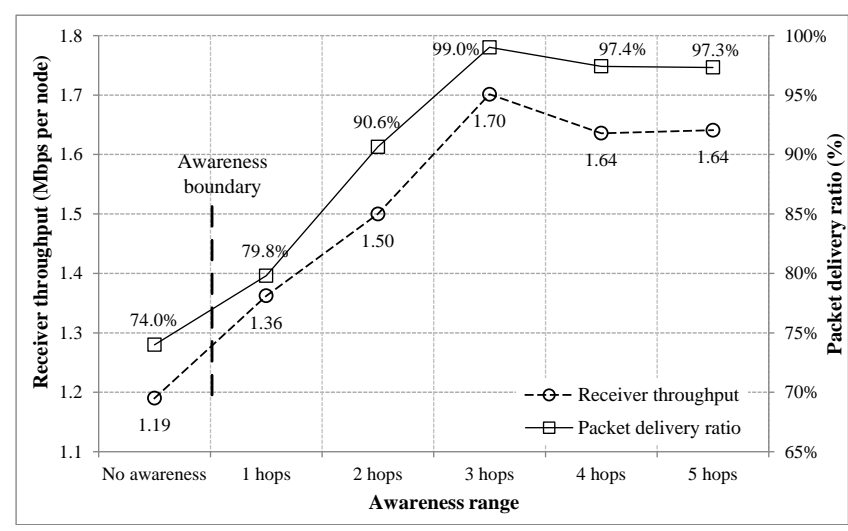

Fig. 2: Receiver throughput and delivery ratio results for different awareness ranges (and 50 slots per TDMA cycle).

node. Latency is only measured for packets that are received successfully. Since we are evaluating the MAC layer only, we have not incorporated retransmission attempts at the application layer, and no Time To Live (TTL) was enforced.

\section{RESULTS}

The results in Fig. 2 illustrate the receiver throughput and delivery ratio for different awareness ranges. The results show that both the packet delivery ratio and receiver throughput are at a minimum for the neighbor-agnostic case, where slots are chosen at random. When the awareness is extended to the immediate neighbors, i.e. a 0-hop awareness range, the packet delivery ratio and receiver throughput both increase. This increase is due to the more informed slot selection. However, the packet delivery ratio is still only $79.8 \%$ due to the hidden terminal problem.

For further increases in awareness range, the performance also increases, up to and including an awareness range of 2 hops. At this point the delivery ratio is almost $99.0 \%$. For awareness ranges above 2 hops, the performance degrades - too many hops results in unnecessary awareness (i.e. awareness of nodes that cannot contend with the node under consideration), which causes SAT saturation and too frequent SAT refreshing (slot re-allocation). In fact, for a higher number of neighbors in the awareness range (not shown), i.e. higher node densities or longer transmission range, the 3-hop and 4hop configurations perform worse than the 0-hop configuration for this reason.

There was no significant change in end-to-end latency, since the TDMA-style structure was the same for all awareness ranges.

The packet delivery ratio results for different TDMA frame sizes and awareness ranges are shown in Fig. 3. The results show that, for the case when 0 hops are used to accumulate SAT information, the packet delivery ratio increases (with a slowing rate of increase) when the TDMA frame size increases. This is because the probability of collisions decreases when more slots are used for the same number of nodes in the awareness range. When the awareness range is extended

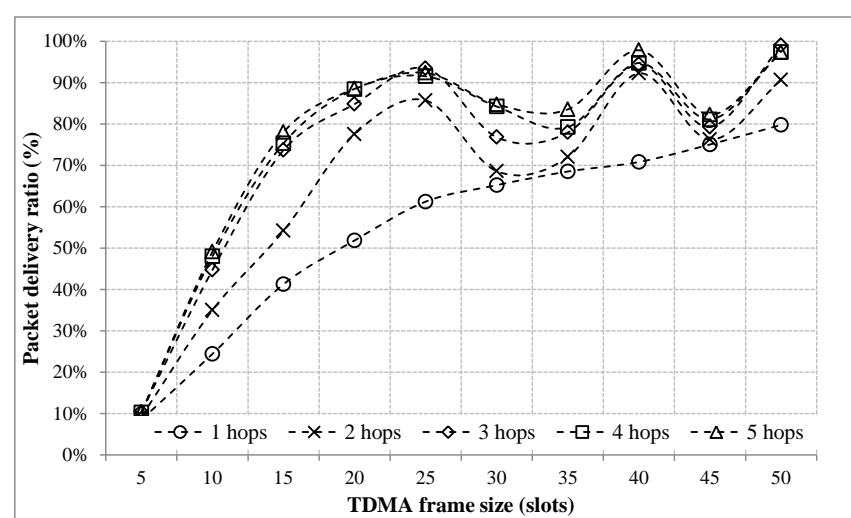

Fig. 3: Packet delivery ratio results.

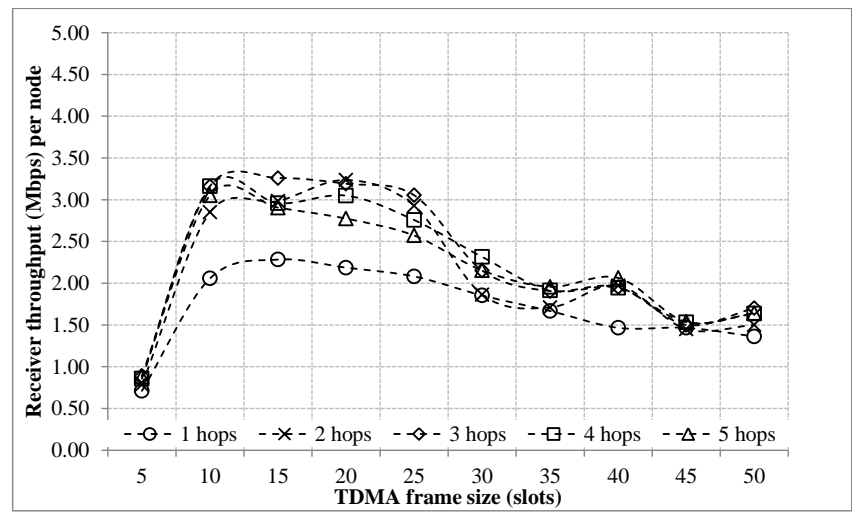

Fig. 4: Receiver throughput results.

to beyond 0 hops, the packet delivery ratio shows oscillatory behavior with respect to the TDMA frame size, with peaks at 20 and 40 slots, and troughs at 30 and 45 slots. Although not shown here, these peaks remain at these locations for different number of neighbors in the awareness range. The oscillations are also evident in the receiver throughput (not transmitter throughput) and latency results, and therefore most likely due the competing concurrent processes: SAT accumulation, SAT saturation, and SAT refresh described in section IV-A.

The receiver throughput results for different TDMA frame

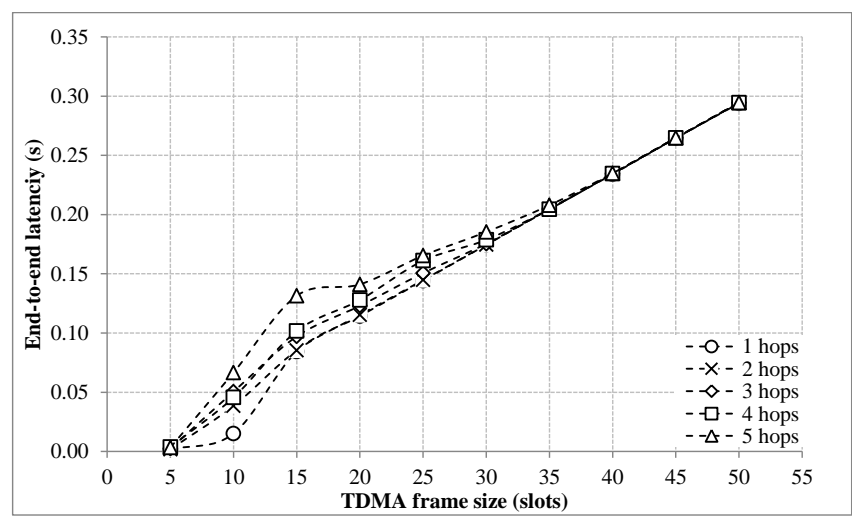

Fig. 5: Latency results. 
sizes and awareness ranges are shown in Fig. 4. The slots in any TDMA-based MAC represent a supply of transmission opportunities, while the number of nodes represents the demand for slots. As the number of slots increases, the demand is satisfied, leading to an increase of receiver throughput. This increase occurs until the number of free slots is higher than the number of requested slots, leading to a reduction in receiver throughput as a result of unused slots. This behavior is noticeable in the 0-hop configuration, where the receiver throughput initially increases as the demand for slots exceeds the number of available free slots. The optimal receiver throughput is obtained with 2 hops, and at 15 slots. Although not shown, simulation tests have shown this peak to be different for different number of neighbors in the awareness range - the number of slots required for optimal performance increases as the number of nodes in the awareness range increases, while the number of hops required decreases for increases in the number of nodes in the awareness range. The results make intuitive sense: For few neighbors, more hops are required to have an awareness range that is big enough to overcome both the static and mobile manifestations of the hidden terminal problem. For more neighbors that can share information and collaboratively cover road area, fewer hops are required.

The latency results for the three neighbor counts are presented in Fig. 5. The results show that the latency values are as expected from a TDMA-based MAC with a fixed frame size, except in the case of many hops $(H>2)$, which leads to SAT refresh, since nodes react to irrelevant (far away) information. Re-selection of slots consumes time, since a node needs to wait a whole cycle before it can re-allocate a slot, which increases the latency. One would expect high levels of slot re-selection at frame sizes of 5 slots and 10 slots too, but due to the high level of interference as a result of too few slots, the conflicting slot selections go unnoticed (which can also be seen from the delivery ratio results). This interference hides the SAT saturation because the transmissions collide, with the result that receiving nodes do not record the SAT information of the owning nodes (i.e. the slots are used and the transmissions unsuccessful), which means the slots will appear available to slot-seeking nodes.

\section{CONCLUSION}

We evaluated the performance of a TDMA-based MAC using metrics such as packet delivery ratio, receiver throughput, and end-to-end latency. The results show that there exists an awareness range (hop count) and that results in optimal receiver throughput and packet delivery ratio. The results further demonstrate that the packet delivery ratio approaches $100 \%$ as TDMA frame size is increased, however, rather than an sigmoidal increase, which is the case for 0-hops, the multihop results show an oscillatory effect, superimposed on the the increase, probably due to the three competing processes in the MAC. Receiver throughput is shown to have an optimal TDMA frame size close to the number of in-range neighbors. The results also demonstrate that the latency increases as the number of slots increase, due to the longer TDMA cycles.
It can be concluded that in networks with high mobility (such as VANET), the awareness range has to be extended to beyond the immediate in-range neighbors, and even beyond the first-hop neighbors to overcome the hidden terminal problem, and collisions caused by node mobility. Awareness beyond a relevance threshold, however, could also affect performance adversely.

\section{REFERENCES}

[1] J. B. Kenney, "Dedicated Short-Range Communications (DSRC) Standards in the United States," Proceedings of the IEEE, vol. 99, no. 7, pp. 1162-1182, July 2011.

[2] P. Papadimitratos, A. La Fortelle, K. Evenssen, R. Brignolo, and S. Cosenza, "Vehicular communication systems: Enabling technologies, applications, and future outlook on intelligent transportation," Communications Magazine, IEEE, vol. 47, no. 11, pp. 84-95, November 2009.

[3] A. Vinel, C. Campolo, J. Petit, and Y. Koucheryavy, "Trustworthy Broadcasting in IEEE 802.11p/WAVE Vehicular Networks: Delay Analysis," Communications Letters, IEEE, vol. 15, no. 9, pp. 1010-1012, September 2011.

[4] M. Torrent-Moreno, D. Jiang, and H. Hartenstein, "Broadcast reception rates and effects of priority access in 802.11-based vehicular ad-hoc networks," in Proceedings of the 1st ACM international workshop on Vehicular ad hoc networks, ser. VANET '04. ACM, 2004, pp. 10-18.

[5] K. Sjöberg, E. Uhlemann, and E. Ström, "How Severe Is the Hidden Terminal Problem in VANETs When Using CSMA and STDMA?" in Vehicular Technology Conference (VTC Fall), 2011 IEEE, September 2011, pp. $1-5$.

[6] IEEE, "IEEE Standard for Information technology - Telecommunications and information exchange between systems Local and metropolitan area networks - Specific requirements Part 11: Wireless LAN Medium Access Control (MAC) and Physical Layer (PHY) Specifications," IEEE P802.11-REVmb/D12, November 2011, pp. 1-2910, 2012.

[7] Y. Bi, K.-H. Liu, L. Cai, X. Shen, and H. Zhao, "A multi-channel token ring protocol for QoS provisioning in inter-vehicle communications," Wireless Communications, IEEE Transactions on, vol. 8, no. 11, pp. 5621-5631, November 2009.

[8] H. Su and X. Zhang, "Clustering-Based Multichannel MAC Protocols for QoS Provisionings Over Vehicular Ad Hoc Networks," Vehicular Technology, IEEE Transactions on, vol. 56, no. 6, pp. 3309-3323, November 2007.

[9] T. Kim, S. Jung, and S. Lee, "CMMP: Clustering-Based Multi-channel MAC Protocol in VANET," in Computer and Electrical Engineering (ICCEE) 2009. Second International Conference on, vol. 1, December 2009, pp. 380-383.

[10] K. Bilstrup, E. Uhlemann, E. G. Ström, and U. Bilstrup, "On the ability of the $802.11 \mathrm{p}$ MAC method and STDMA to support real-time vehicle-to-vehicle communication," EURASIP Journal of Wireless Communications Networks, pp. 5:1-5:13, Jan. 2009. [Online]. Available: http://dx.doi.org/10.1155/2009/902414

[11] F. Yu and S. Biswas, "A Self Reorganizing MAC Protocol for Intervehicle Data Transfer Applications in Vehicular Ad Hoc Networks," in Information Technology (ICIT), 2007. 10th International Conference on, December 2007, pp. 110-115.

[12] N. Lu, Y. Ji, F. Liu, and X. Wang, "A Dedicated Multi-Channel MAC Protocol Design for VANET with Adaptive Broadcasting," in Wireless Communications and Networking Conference (WCNC), 2010 IEEE, April 2010, pp. 1-6.

[13] H. Cozzetti and R. Scopigno, "RR-Aloha+: A slotted and distributed MAC protocol for vehicular communications," in Vehicular Networking Conference (VNC), 2009 IEEE, Oct. 2009, pp. 1 -8.

[14] C. Sommer, R. German, and F. Dressler, "Bidirectionally Coupled Network and Road Traffic Simulation for Improved IVC Analysis," IEEE Transactions on Mobile Computing, vol. 10, no. 1, pp. 3-15, January 2011.

[15] M. Boban, O. Tonguz, and J. Barros, "Unicast communication in vehicular ad hoc networks: a reality check," Communications Letters, IEEE, vol. 13, no. 12, pp. 995-997, December 2009.

[16] M. J. Booysen, S. Zeadally, and G.-J. van Rooyen, "Performance comparison of media access control protocols for vehicular ad hoc networks," Networks, IET, vol. 1, no. 1, pp. 10-19, March 2012. 